\title{
Deep vein thrombosis is accurately predicted by comprehensive analysis of the levels of microRNA-96 and plasma D-dimer
}

\author{
XUESHENG XIE, CHANGPENG LIU, WEI LIN, BAOMING ZHAN, CHANGJUN DONG, \\ ZHEN SONG, SHILEI WANG, YINGGUO QI, JIALI WANG and ZENGQUAN GU \\ Department of Orthopedics, Laiwu People's Hospital, Laiwu, Shandong 271100, P.R. China
}

Received December 22, 2014; Accepted January 21, 2016

DOI: $10.3892 /$ etm. 2016.3546

\begin{abstract}
The aim of the present study was to investigate the association between platelet microRNA-96 (miR-96) expression levels and the occurrence of deep vein thrombosis (DVT) in orthopedic patients. A total of consecutive 69 orthopedic patients with DVT and 30 healthy individuals were enrolled. Ultrasonic color Doppler imaging was performed on lower limb veins after orthopedic surgery to determine the occurrence of DVT. An enzyme-linked fluorescent assay was performed to detect the levels of D-dimer in plasma. A quantitative polymerase chain reaction assay was performed to determine the expression levels of miR-96. Expression levels of platelet miR-96 were significantly increased in orthopedic patients after orthopedic surgery. miR-96 expression levels in orthopedic patients with DVT at days 1, 3 and 7 after orthopedic surgery were significantly increased when compared with those in the control group. The increased miR-96 expression levels were correlated with plasma D-dimer levels in orthopedic patients with DVT. However, for the orthopedic patients in the non-DVT group following surgery, miR-96 expression levels were correlated with plasma D-dimer levels. In summary, the present results suggest that the expression levels of miR-96 may be associated with the occurrence of DVT. The occurrence of DVT may be accurately predicted by comprehensive analysis of the levels of miR-96 and plasma D-dimer.
\end{abstract}

\section{Introduction}

Deep vein thrombosis (DVT) is a severe postoperative complication, which commonly occurs in $40-80 \%$ of patients undergoing orthopedic surgeries, such as total hip replacement, total knee replacement, and hip fractures surgery (1-3). Pulmonary embolism, a potentially life-threatening disease

Correspondence to: Dr Zengquan Gu, Department of Orthopedics, Laiwu People's Hospital, 1 Xuehu Street, Laiwu, Shandong 271100, P.R. China

E-mail: gzq222@126.com

Key words: orthopedic surgery, deep vein thrombosis, microRNA-96 with a high mortality rate in China, may result from a detached thrombus that travels to the lungs (4).

Formation of thrombus is closely related to the activation of platelets $(5,6)$. Under the action of coagulation factor, platelets are activated and aggregated, eventually forming a thrombus (7). Vesicle-associated membrane protein 8 (VAMP8) is a vesicle-associated major membrane protein of soluble N-ethylmaleimide-sensitive factor attachment protein receptor (8). VAMP8 is necessary for platelet activation, which is involved in the process of platelet degranulation $(9,10)$. A previous study showed that increased expression of microRNA-96 (miR-96) lead to a reduction in platelet activation by inhibiting the expression of VAMP8 protein (11). It has been suggest that the occurrence of DVT may be predicted by detection of miR-96 expression in platelets (12). D-dimer is a fibrin degradation product in blood after a thrombus is degraded by thrombin, coagulation factor XIIIa and fibrinolysin (13-15). Levels of D-dimer may reflect the activity of coagulation and the fibrinolytic system, which may be used for diagnosis of DVT (16). However, the correlation between miR-96 expression and D-dimer in the development of DVT is unknown.

In the present study, levels of miR-96 expression in platelets were detected in patients with orthopedic conditions following surgery. The association between miR-96 expression and occurrence of DVT was thus determined. Furthermore, the correlation between miR-96 expression in platelets and of D-dimer levels in plasma was investigated.

\section{Materials and methods}

Patients. A total of consecutive 8 patients with DVT after orthopedic surgery (DVT group; 3 males and 5 females) were enrolled in this study in Laiwu People's Hospital (Laiwu, China). The age of these patients ranged between 51 and 68 years, with an average age of 63.4 years. The prognosis of DVT referred to the clinical manifestations, imaging and laboratory test results, including whether deep vein thrombosis, limb swelling, thickened circumference, pain or tenderness, superficial vein dilation, skin pigmentation and/or abnormal D-dimer values were detected. In the corresponding period, a total of consecutive 61 orthopedic patients without DVT following orthopedic surgery (non-DVT group) were enrolled in this study. These patients included 35 males and 26 females, with ages ranging between 31 and 72 years, and 
an average age of 58.3 years. As a control, a total of consecutive 30 healthy individuals (control group) were enrolled in this study, which included 15 males and 15 females. The age of these healthy individuals ranged between 30 and 88 years, with an average age of 56.5 years.

The exclusion criteria of DVT group were as follows: i) Patients with DVT before orthopedic surgeries; ii) patients with active bleeding and spontaneous intracranial hemorrhage recently; iii) patients with bleeding disorders and undergoing anticoagulant treatment; iv) patients with severe liver and kidney disease; v) patients had not undergone surgery and trauma; vi) patients had history of thrombosis; and vii) patients with malignancies, inflammatory diseases. Prior written and informed consent were obtained from every patient. The study was approved by the ethics review board of Laiwu People's Hospital.

Color Doppler ultrasonography. The lower limb veins were detected in patients before orthopedic surgery and at days 3 and 7 after orthopedic surgery. Venous diameter, blood flow, intraluminal pressure, deep venous valve function and thrombosis formation were determined using a color Doppler ultrasonography imaging system (ACUSON Antares Color Doppler Ultrasound System; Siemens AG, Berlin, German).

Purification of platelets. Peripheral blood was extracted from patients after orthopedic surgery and from healthy individuals. The samples were mixed with $85 \mathrm{mM}$ trisodium citrate, $78 \mathrm{mM}$ citric acid and $111 \mathrm{mM}$ glucose (all from Sigma-Aldrich, St. Louis, MO. USA). Following centrifugation at $80 \mathrm{x} \mathrm{g}$ for $10 \mathrm{~min}$, the mixtures were mixed with $2 \mathrm{mM}$ ethylenediaminetetraacetic acid (EDTA; pH 8.0; Sigma-Aldrich) and centrifuged at $1,000 \mathrm{x} \mathrm{g}$ for $10 \mathrm{~min}$. The pellets of platelets were resuspended in $3 \mathrm{ml}$ Beads Buffer [0.8\% NaCl, 0.02\% $\mathrm{KCl}, 0.144 \% \mathrm{Na}_{2} \mathrm{HPO}_{4}, 0.024 \% \mathrm{KH}_{2} \mathrm{PO}_{4}, 0.5 \%$ bovine serum albumin and 2 mM EDTA ( $\mathrm{pH} 8.0$ )], then $40 \mu \mathrm{l}$ human CD45 MicroBeads reagent (Miltenyi Biotec GmbH, Bergisch Gladbach, German) was added and mixed gently. The platelets were separated using a MACS ${ }^{\circledR}$ magnetic cell sorting system (Miltenyi Biotec $\mathrm{GmbH}$ ). The purity of platelets was $>99.99 \%$.

Quantitative polymerase chain reaction ( $q P C R)$ assay. Total RNA of platelets were extracted using TRIzol reagent (Invitrogen; Thermo Fisher Scientific, Inc., Carlsbad, CA, USA). RNA quality was detected by RNA electrophoresis and spectrophotometer at an optical density of 260/280 nm. All RNA was reverse transcribed into cDNA using a PrimeScript RT Regent kits (Takara Bio, Inc., Otsu, Japan) according to the manufacturer's protocol. Quantitative PCR assay was conducted using a SYBR PrimeScript RT-PCR kit II (Takara Bio, Inc.) in accordance with the manufacturer's protocol. $\beta$-actin was used as an internal control for miR-96. The primers for miR-96 were as follows: 5'-TTTGGCACTAGC ACATT-3' and 5'-GAGCAGGCTGGAGAA-3'. The primers for $\beta$-actin were: 5'-CATGTACGTTGCTATCCAGGC-3' and 5'-CTCCTTAATGTCACGCACGAT-3'. Oligonucleotide primers of miR-96 and $\beta$-actin were synthesized by Invitrogen (Thermo Fisher Scientific, Inc.). The $20 \mu \mathrm{l}$ reaction system consisted of $0.5 \mu \mathrm{l}$ cDNA, $10 \mu \mathrm{l}$ SYBR Ex Taq, $0.5 \mu 1$

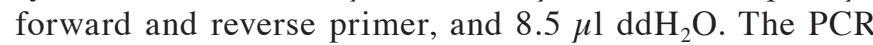

thermocycling conditions were as follows: Denaturation at $95^{\circ} \mathrm{C}$ for $10 \mathrm{sec}, 95^{\circ} \mathrm{C}$ for $5 \mathrm{sec}$, and $60^{\circ} \mathrm{C}$ for $20 \mathrm{sec}$ for a total of 40 cycles. For each sample, PCR reaction was repeated for at least 3 times. The gene expression levels were calculated with the $2^{-\Delta \Delta \mathrm{Cq}}$ method.

Western blot analysis. Total proteins of platelets were extracted using a total protein extraction reagent (BioTeke Corporation, Beijing, China). Total proteins $(100 \mu \mathrm{l})$ were separated on $12 \%$ SDS-PAGE gel, and transferred onto a polyvinylidene fluoride membrane (KLH Bioscience, Shanghai, China). After blocking with $5 \%$ non-fat milk in Tris-buffered saline with Tween-20 at $37^{\circ} \mathrm{C}$ for $2 \mathrm{~h}$, the membrane was incubated at $4^{\circ} \mathrm{C}$ for $24 \mathrm{~h}$ with rabbit anti-human VAMP8 polyclonal primary antibodies (1:800; cat. no. ab76021; Abcam, Cambridge, MA, USA). The membrane was subsequently incubated at $37^{\circ} \mathrm{C}$ for $1 \mathrm{~h}$ with horse radish peroxidase-conjugated goat anti-rabbit secondary antibodies (1:2,000; cat. no. ab181658; Abcam). $\beta$-tubulin was used as an internal control. Blots were detected using ECL Plus enhanced chemiluminescence reagent (EMD Millipore, Billerica, MA, USA). Image quantifications were performed using ImageLab software version 4.1 (Bio-Rad Laboratories, Inc., Hercules, CA, USA). Experiments were repeated at least 3 times.

Plasma D-dimer detection. Peripheral venous blood samples were collected from patients at days 1,3 and 7 after orthopedic surgery. For control, peripheral venous blood samples were collected from healthy individuals. Subsequently, $\sim 1.8-\mathrm{ml}$ samples were transferred into anticoagulant tube with $0.2 \mathrm{ml}$ citric acid $\left(10^{9} \mathrm{mM}\right)$ and mixed. The mixtures were centrifuged at $400 \mathrm{x} \mathrm{g}$ for $15 \mathrm{~min}$. The plasma supernatants were finally transferred to a new tube for the analysis of D-dimer. D-dimer was detected by enzyme-linked fluorescent assay using a VIDAS instrument and VIDAS D-dimer Exclusion II kit (VIDAS S60; BioMerieux, Inc., Grenoble, France), in accordance with the manufacturer's instructions.

Statistical analysis. All results are expressed as the mean \pm standard deviation. All statistical analyses were performed with SPSS software, version 13.0 for Windows (SPSS, Inc., Chicago, IL, USA). Paired $t$-test was used to analyze comparisons between groups and analysis of paired data. Pearson correlation was used to analyze the association between miR-96 expression and plasma D-dimer levels for the prediction of DVT. $\mathrm{P}<0.05$ was considered to indicate a statistically significant difference.

\section{Results}

Diagnosis of patients with DVT by color Doppler ultrasonography. To determine the occurrence of DVT, ultrasonic color Doppler imaging was performed on the lower limb veins of patients and healthy individuals. As shown in Fig. 1A, for the non-DVT group, there was no sign of thrombus in the femoral vein. However, for the DVT group, coronal imaging showed significant vein stenosis and several thrombi in the femoral vein (Fig. 1B). These results suggest that occurrence of DVT can be clearly diagnosed using color Doppler ultrasonography. 

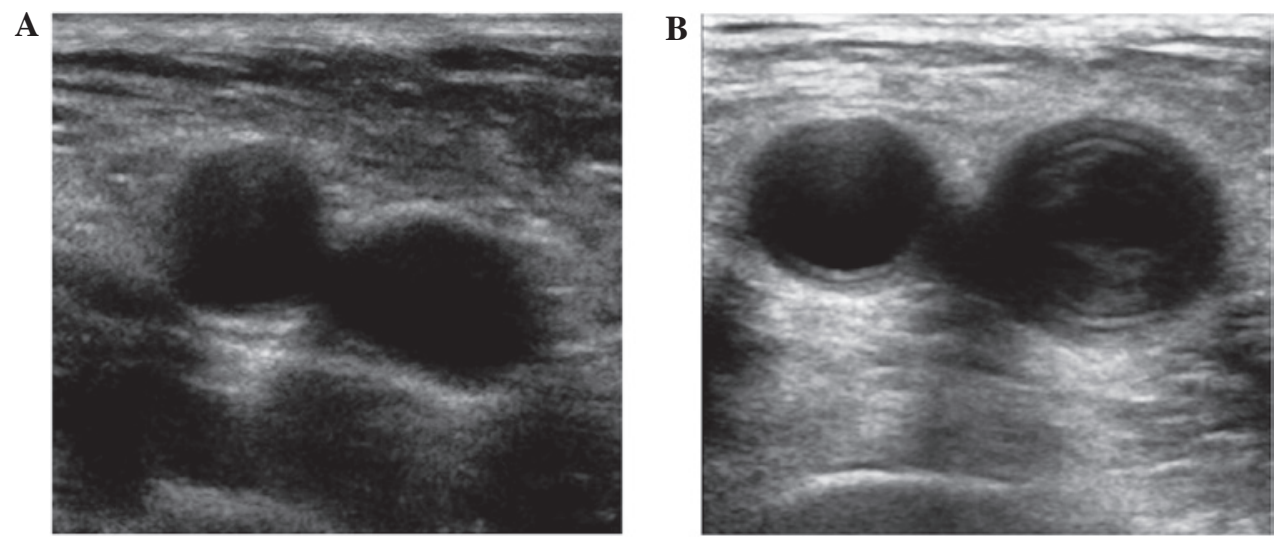

Figure 1. Color Doppler ultrasonography imaging between deep vein thrombosis (DVT) and non-DVT groups. Color Doppler ultrasonography was performed to detect the size, localization, echogenicity, margins and tendency of the thrombus structure. (A) Coronal imaging showed that (A) non-significant stenosis occurred in the femoral vein of the non-DVT group and that (B) several thrombi and venous stenosisoccurred in the femoral vein of the DVT group.

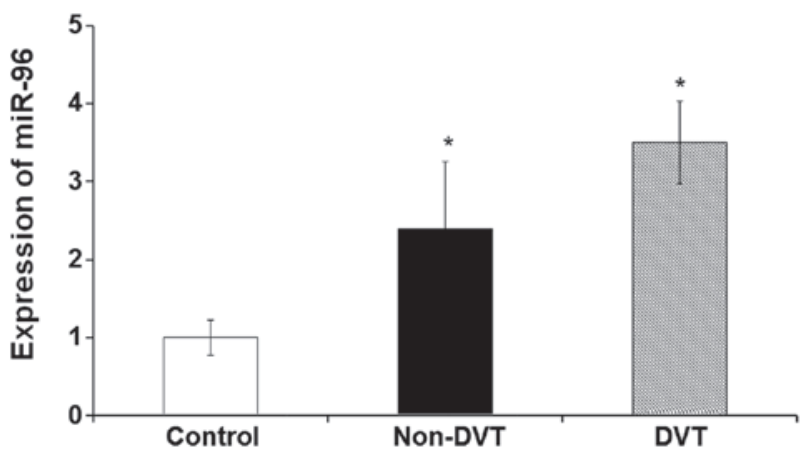

Figure 2. Expression levels of miR-96 in patients with orthopedic disease and healthy individuals. Expression levels of miR-96 were detected using a quantitative polymerase chain reaction assay. Experiments were repeated at least 3 times. U6 was used as an internal control for miR-96. Histograms showed mean relative expression levels of miR-96 in the control, non-DVT and DVT groups. ${ }^{*} \mathrm{P}<0.05$ vs. control group. miR-96, microRNA-96; DVT, deep vein thrombosis.

Expression levels of platelet miR-96 are significantly increased in patients following orthopedic surgery. To determine the expression levels of miR-96 between patients with orthopedic disease and healthy individuals, a qPCR assay was performed. As shown in Fig. 2, expression levels of platelet miR-96 in the DVT and non-DVT group were significantly increased compared with the control group $(\mathrm{P}<0.05)$. The expression levels of miR-96 in the DVT group were higher than those in the non-DVT group. However, there was no significant difference between the DVT and the non-DVT group. These results indicate that the expression levels of platelet miR-96 are significantly increased in orthopedic patients after orthopedic surgery. High expression of miR-96 may be associated with the occurrence of DVT.

Expression levels of VAMP8 are increased in the DVT group and reduced in the non-DVT group. To investigate the expression levels of the platelet-related protein VAMP8 between orthopedic patients and healthy individuals, western blot analysis was performed. As shown in Fig. 3, the expression levels of VAMP8 in the non-DVT group were significantly decreased when compared with those in the control group $(\mathrm{P}<0.05)$. However, the expression levels of VAMP8 in the
A

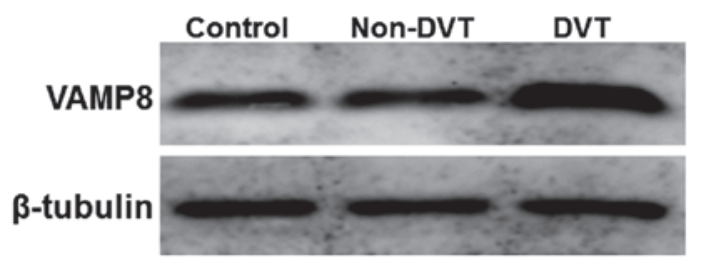

B

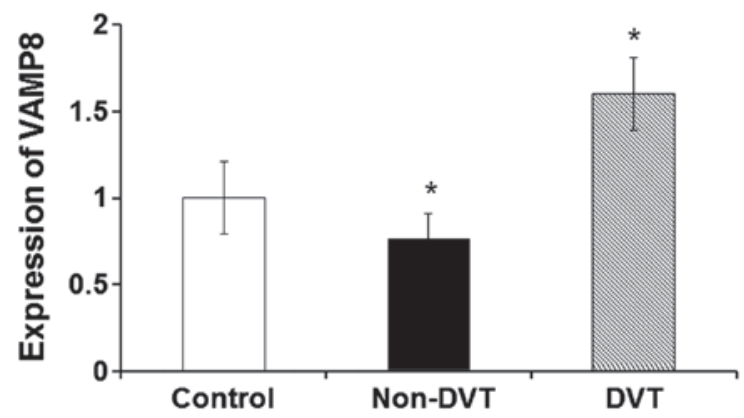

Figure 3. Expression levels of VAMP8 in patients with orthopedic disease and healthy individuals, as analyzed by western blot analysis. $\beta$-tubulin was used as a loading control. (A) Representative western blotting results. (B) Quantitative western blotting results. ${ }^{*} \mathrm{P}<0.05$ vs. control group. DVT, deep vein thrombosis; VAMP8, vesicle-associated membrane protein 8 .

DVT group were significantly increased when compared with those in the control group $(\mathrm{P}<0.05)$. These results indicate that the expression levels of VAMP8 are increased in the DVT group while decreased in the non-DVT group. Expression of VAMP8 in platelets may be inhibited by the high expression of miR-96 in the non-DVT group.

miR-96 expression levels are increased in the non-DVT group, but are not correlated with plasma D-dimer levels. To investigate the association between miR-96 expression levels and plasma D-dimer levels in the non-DVT group, Pearson correlation analysis was performed. The expression levels of miR-96 were detected using a qPCR assay in the non-DVT group at days 1 , 3 and 7 after orthopedic surgery. For control, the expression levels of miR-96 in the control group were detected. As shown in Fig. 4A, miR-96 expression levels in the non-DVT group at days 1,3 and 7 after orthopedic surgery were significantly 

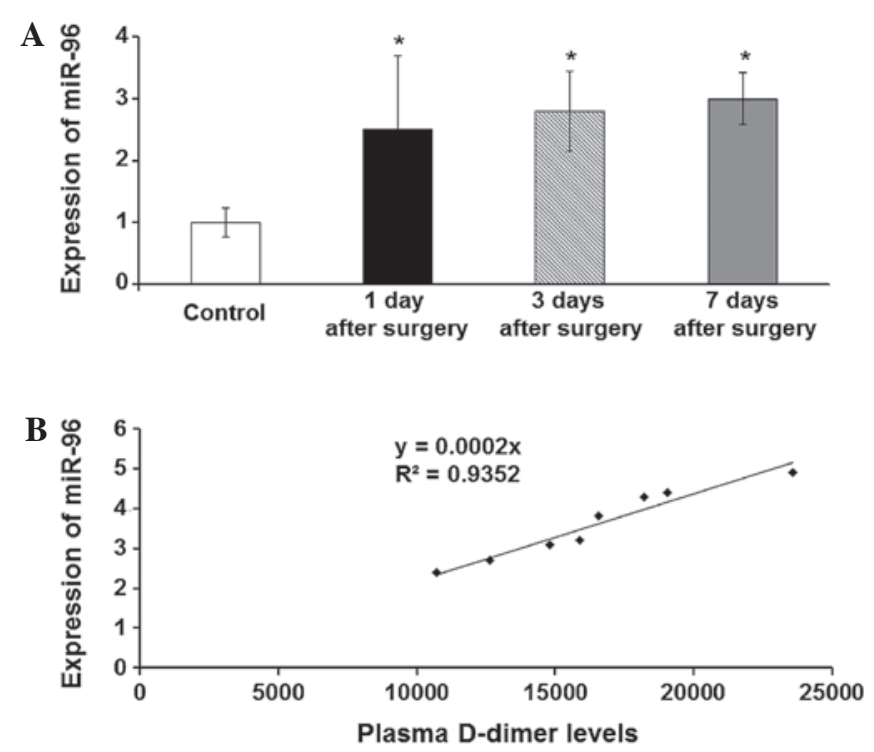

Figure 4. Association between platelets miR-96 expression and plasma D-dimer levels in the non-deep vein thrombosis (DVT) group. The expression levels of miR-96 were detected using a quantitative polymerase chain reaction assay in the non-DVT group at days 1, 3 and 7 after orthopedic surgery. Levels of plasma D-dimer were detected at the corresponding time points using an enzyme-linked fluorescent assay. The association between miR-96 expression and plasma D-dimer levels was analyzed using Pearson correlation analysis. (A) Histograms show mean normalized miR-96 expression levels in the control and non-DVT groups at days 1, 3 and 7 after orthopedic surgery. Error bars show standard error of the mean. "P<0.05 vs. control group. (B) Correlation between platelets miR-96 expression and plasma D-dimer levels in the non-DVT group. miR-96, microRNA-96.

increased when compared with those in the control group $(\mathrm{P}<0.05)$. Pearson correlation analysis results showed that the gradually increased miR-96 expression levels were not linearly correlated with plasma D-dimer levels in the non-DVT group (Fig. 4B). Levels of plasma D-dimer are commonly used for the prediction of DVT (17). These results suggest although miR-96 expression levels are increased in the non-DVT group, miR-96 expression levels are not correlated with plasma D-dimer levels.

Increased miR-96 expression levels are correlated with plasma $D$-dimer levels in the DVT group. To investigate whether miR-96 expression levels were correlated with plasma D-dimer levels in the DVT group, Pearson correlation analysis were performed. In the DVT group at days 1,3 and 7 after orthopedic surgery, the expression levels of miR-96 were detected using a qPCR assay. As shown in Fig. 5A, miR-96 expression levels in the DVT group at days 1,3 and 7 after orthopedic surgery were significantly increased when compared with those in the control group $(\mathrm{P}<0.05)$. Pearson correlation analysis results showed that miR-96 expression levels were correlated with plasma D-dimer levels in the DVT group (Fig. 5B). These results indicate that the occurrence of DVT may be predicted by comprehensive analysis of the levels of miR-96 and plasma D-dimer.

\section{Discussion}

DVT is a common postoperative complication in orthopedic patients, accounting for $\sim 50 \%$ of the total patients with embolization (18). Pulmonary embolism caused by DVT is a potentially life-threatening disease with a high mortality rate
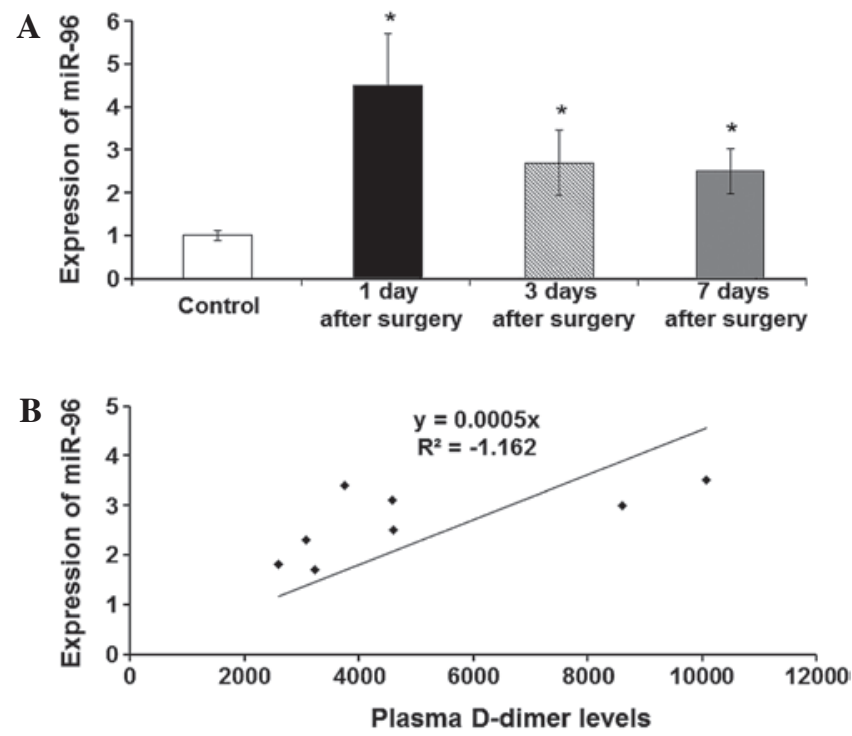

Figure 5. Association between platelet miR-96 expression and plasma D-dimer levels in the deep vein thrombosis (DVT) group. The expression levels of miR-96 were detected using a quantitative polymerase chain reaction assay in the DVT group at days 1,3 and 7 after orthopedic surgery. Levels of plasma D-dimer were detected at the corresponding time points using an enzyme-linked fluorescent assay. The association between miR-96 expression and plasma D-dimer levels was analyzed by Pearson correlation analysis. (A) Histograms showed mean normalized miR-96 expression levels in the control and DVT groups at days 1, 3 and 7 after orthopedic surgery. Error bars show standard error of the mean. * $\mathrm{P}<0.05$ vs. control group. (B) Correlation between platelets miR-96 expression and plasma D-dimer levels in the DVT group. miR-96, microRNA-96.

of $0.5 \%$ (4). Therefore, early prevention and accurate diagnosis are key to reducing morbidity and mortality.

As an important miRNA in platelets, miR-96 inhibits the expression of VAMP8, which serves a crucial function in reducing the activation of platelets (11). In the present study, the expression levels of miR-96 and VAMP8 were determined in the DVT, non-DVT and control groups. The results showed that in the non-DVT group, miR-96 expression levels were increased while VAMP8 expression levels were decreased when compared with those in the control group. For the DVT group, miR-96 expression levels were also significantly increased in platelets. The expression levels of miR-96 in the DVT group were higher than those in the non-DVT group. However, VAMP8 expression levels were not decreased in the DVT group, as compared with the non-DVT group. These results indicate that the expression of VAMP8 after orthopedic surgery may be regulated by miR-96, in addition to other signaling pathways. Plasma D-dimer levels indicate the activity of the coagulation/fibrinolytic system in vivo, which can be used as a marker for the prediction of DVT.

The present results showed that miR-96 expression levels in the DVT group at days 1, 3 and 7 after orthopedic surgery were significantly increased when compared with those in the control group. The increased miR-96 expression levels were correlated with plasma D-dimer levels in the DVT group. However, although miR-96 expression levels were increased in the non-DVT group, miR-96 expression levels were not correlated with plasma D-dimer levels. Typically, due to the stress responses, compared with normal subjects the levels of various proteins and factors would be elevated in the patients 
following surgery. However, the single factor detection may not be sufficient for disease prediction. The results of the present study demonstrated that the expression levels of miR-96 in the patients following surgery were elevated in both the DVT and non-DVT groups. Compared with the DVT group, the D-dimer value was also increased in the DVT group, in addition to elevated miR-96 expression levels, indicating an association between the two events in these patients. Based on these findings, the simultaneous detection of elevated miR-96 expression levels and D-dimer values could contribute to a more accurate prediction of the incidence of DVT.

In conclusion, the expression levels of platelets miR-96 are significantly increased in patients after orthopedic surgery. The expression levels of platelets miR-96 are correlated with the levels of plasma D-dimer in the DVT group. These results suggest that the occurrence of DVT may be accurately predicted by comprehensive analysis with levels of miR-96 and plasma D-dimer.

\section{Acknowledgements}

The authors thank Professor Xuesheng Sun, Mr. Yichen Dong and Ms. Huiyun Zhang from Department of Orthopedics of Laiwu People's Hospital (Laiwu, China).

\section{References}

1. Dua A, Neiva S and Sutherland A: Does previous varicose vein surgery alter deep vein thrombosis risk after lower limb arthroplasty? Orthop Surg 4: 222-2226, 2012.

2. Shah K, Thevendran G, Younger A and Pinney SJ: Deep-vein thrombosis prophylaxis in foot and ankle surgery: What is the current state of practice? Foot Ankle Spec 8: 101-116, 2015.

3. Rahman JR, Magan A and Tytherleigh-Strong GM: Subclavian vein thrombosis following acute internal fixation of a clavicular fracture. Shoulder Elb 5: 108-110, 2013.

4. Wu O, Clark P, Lowe GD, Walker ID and Greer IA; Thrombosis: Risk and Economic Assessment of Thrombophilia Screening (TREATS) Study Group: Thrombophilia and venous thromboembolism after total hip or knee replacement surgery: A systematic review. J Thromb Haemost 3: 811-813, 2005.

5. Silverstein RL: Platelet CD36 links not only $\alpha$-granule-derived proteins to thrombus stability but also metabolic and oxidant stress to a prothrombotic phenotype. Arterioscler Thromb Vasc Biol 34: 1120-1121, 2014.
6. Kuijpers MJ, de Witt S, Nergiz-Unal R, van Kruchten R, Korporaal SJ, Verhamme P, Febbraio M, Tjwa M, Voshol PJ, Hoylaerts MF, et al: Supporting roles of platelet thrombospondin-1 and CD36 in thrombus formation on collagen. Arterioscler Thromb Vasc Biol 34: 1187-1192, 2014.

7. Heestermans M, Salloum-Asfar S, Salvatori D, Laghmani EH, Luken BM, Zeerleder SS, Spronk HM, Korporaal SJ, Wagenaar GT, Reitsma PH and van Vlijmen BJ: Role of platelets, neutrophils, and factor XII in spontaneous venous thrombosis in mice. Blood [Epub ahead of print], 2016.

8. Marshall MR, Pattu V, Halimani M, Maier-Peuschel M, Müller ML, Becherer U, Hong W, Hoth M, Tschernig T, Bryceson YT and Rettig J: VAMP8-dependent fusion of recycling endosomes with the plasma membrane facilitates $\mathrm{T}$ lymphocyte cytotoxicity. J Cell Biol 210: 135-151, 2015.

9. Polgár J, Chung SH and Reed GL: Vesicle-associated membrane protein 3 (VAMP-3) and VAMP-8 are present in human platelets and are required for granule secretion. Blood 100: 1081-1083, 2002.

10. Ren Q, Barber HK, Crawford GL, Karim ZA, Zhao C, Choi W, Wang CC, Hong W and Whiteheart SW: Endobrevin/VAMP-8 is the primary v-SNARE for the platelet release reaction. Mol Biol Cell 18: 24-33, 2007.

11. Kondkar AA, Bray MS, Leal SM, Nagalla S, Liu DJ, Jin Y, Dong JF, Ren Q, Whiteheart SW, Shaw C and Bray PF: VAMP8/endobrevin is overexpressed in hyperreactive human platelets: Suggested role for platelet microRNA. J Thromb Haemost 8: 369-378, 2010.

12. Zhu HQ: Differential expression of miR-223 and miR-96 in platelets and its association with clopidogrel reactivity in patients with coronary heart disease. Cardiovascular Disease Journal Of integrated traditional Chinese and Western Medicine 1: 7-8, 2015 (In Chinese).

13. Molugu C, Fisher G, Hirons B, Hughes D and Raftery S: P151 V-DimERS study-value of D-Dimers in estimating risk of significant pulmonary embolism and deep vein thrombosis. Thorax 68 (Suppl 3): A144-A144, 2013.

14. Wells P: Overview and comparison of D-dimer assay kits for DVT and PE. Clin Adv Hematol Oncol 2: 160, 2004

15. Wedlund A and Voslar A: Is the D-dimer test a viable option for the detection of PE? J Nucl Med 55 (Suppl 1): 2708, 2014.

16. Lees D, Griffiths P, Paxton C and Wahbi Z: Can D-dimer assay, together with clinical probability predict computed tomography pulmonary angiogram (CTPA) outcomes for pulmonary embolism (PE)? Eur Respir J 38 (Suppl 55): 582, 2011.

17. Hodgkiss-Harlow KD and Betti K: Institutional quality outcome measures: Investigating the impact of raising the positive D-dimer threshold for DVT as confirmed by duplex ultrasound. J Vasc Surg 62: 527, 2015.

18. Shulman RM, Buchan C, Bleakney RR and White LM: Low prevalence of unexpected popliteal DVT detected on routine MRI assessment of the knee. Clin Imaging 40: 79-85, 2016. 